\title{
Clinical value of dual-phase F-18 sodium fluoride PET/CT for diagnosing bone metastasis in cancer patients with solitary bone lesion
}

\author{
Jeong Won Lee ${ }^{1 \#} \wedge$, Yong-Jin Park ${ }^{2 \#} \wedge$, Youn Soo Jeon ${ }^{3} \wedge$, Ki Hong Kim ${ }^{3} \wedge$, Jong Eun Lee ${ }^{4}$, Sung Hoon Hong ${ }^{4} \wedge$, \\ Sang Mi Lee ${ }^{5}$, Su Jin Jang ${ }^{6}$ \\ ${ }^{1}$ Department of Nuclear Medicine, Catholic Kwandong University College of Medicine, International St. Mary's Hospital, Incheon, Korea; \\ ${ }^{2}$ Department of Nuclear Medicine, Samsung Medical Center, Seoul, Korea; ${ }^{3}$ Department of Urology, ${ }^{4}$ Department of Surgery, ${ }^{5}$ Department of \\ Nuclear Medicine, Soonchunhyang University Cheonan Hospital, Cheonan, Korea; ${ }^{6}$ Department of Nuclear Medicine, CHA Bundang Medical \\ Center, CHA University, Seongnam-si, Korea
}

"These authors contributed equally to this work.

Correspondence to: Sang Mi Lee. Soonchunhyang University Cheonan Hospital, 31 Suncheonhyang 6-gil, Dongnam-gu, Cheonan, Chungcheongnamdo 31151, Korea. Su Jin Jang, CHA Bundang Medical Center, CHA University, 59 Yatap-ro, Bundang-gu, Seongnam-si, Gyeonggi-do 13496, Korea. Email: gareen@naver.com; jsjnm07@cha.ac.kr.

Background: The present study aimed to investigate whether dual-phase F-18 sodium-fluoride $(\mathrm{NaF})$ positron emission tomography/computed tomography (PET/CT) could improve the diagnostic accuracy of detecting bone metastasis in cancer patients with a solitary bone lesion compared to conventional F-18 NaF $\mathrm{PET} / \mathrm{CT}$.

Methods: We retrospectively enrolled 113 cancer patients who underwent dual-phase F-18 NaF PET/ CT for the differential diagnosis of a solitary bone lesion seen on bone scintigraphy. According to the dualphase PET/CT protocol, an early-phase scan was acquired immediately after radiotracer injection and a conventional F-18 NaF PET/CT scan was performed. The diagnostic abilities of the visual analysis of conventional and dual-phase PET/CT scans and two quantitative parameters (lesion-to-blood pool uptake ratio on early-phase scan and lesion-to-bone uptake ratio on conventional scan) for detecting bone metastasis were compared. The final diagnosis of bone metastasis was made by histopathological confirmation or follow-up imaging studies.

Results: A metastatic bone lesion was diagnosed in 28 patients (24.8\%). The sensitivity, specificity, and accuracy were $100.0 \%, 70.6 \%$, and $77.9 \%$, respectively, for visual analysis of conventional F-18 NaF PET/CT, 92.9\%, 42.4\%, 54.9\%, respectively, for lesion-to-bone uptake ratio, 96.4\%, 88.2\%, and 90.3\%, respectively, for visual analysis of dual-phase PET/CT, and 92.9\%, 81.2\%, and 83.2\%, respectively, for lesion-to-blood pool uptake ratio. Visual analysis of dual-phase PET/CT was shown to have the highest area under the receiver operating characteristic curve value $(0.923 ; 95 \%$ CI, 0.858-0.965) among all parameters.

Conclusions: Dual-phase F-18 NaF PET/CT showed a high diagnostic ability for detecting bone metastasis with improved specificity and accuracy compared to conventional F-18 NaF PET/CT in cancer patients. Dual-phase F-18 NaF PET/CT might help diagnose bone metastasis in patients with malignancies who were shown to have a solitary bone lesion on bone scintigraphy.

Keywords: Bone; diagnosis; F-18 Sodium-fluoride (NaF); metastasis; positron emission tomography (PET)

^ ORCID: Jeong Won Lee, 0000-0002-2697-3578; Yong-Jin Park, 0000-0002-9305-1274; Youn Soo Jeon, 0000-0003-2730-8585; Ki Hong Kim, 0000-0001-8009-7145; Sung Hoon Hong, 0000-0002-3279-3581; Sang Mi Lee, 0000-0002-7943-3807; Su Jin Jang, 0000-0001-61036830 . 
Submitted Apr 23, 2020. Accepted for publication Jul 17, 2020.

doi: 10.21037/qims-20-607

View this article at: http://dx.doi.org/10.21037/qims-20-607

\section{Introduction}

F-18 Sodium-fluoride $(\mathrm{NaF})$ is a widely used bonespecific imaging radiotracer for positron emission tomography (PET) (1). Although F-18 NaF has a similar pharmacological behavior to that of Tc-99m labeled diphosphonate agents used for bone scintigraphy (1-4), F-18 NaF has superior pharmacokinetic characteristics for bone imaging including higher bone uptake, faster blood clearance and lower protein binding (3-6). Therefore, F-18 NaF PET was shown to have a significantly higher diagnostic accuracy for detecting bone metastasis than bone scintigraphy using Tc-99m labeled diphosphonate agents in patients with diverse malignant diseases (7-9). However, nonmetastatic bone lesions such as degenerative, traumatic, and inflammatory bone lesions can also show increased F-18 $\mathrm{NaF}$ uptake, which are considered false-positive findings in the detection of bone metastasis, thereby limiting its specificity $(6,10)$. Consequently, in contrast to the sensitivity which was consistently high in previous studies, inconsistent results have been found regarding the specificity of F-18 $\mathrm{NaF}$ PET/CT (10-12).

A dual-phase F-18 NaF PET/CT imaging protocol was recently used to enhance the diagnostic ability in various bone and joint diseases (2,13-15). In the dual-phase PET/ CT imaging protocol, early-phase PET/CT imaging is acquired within 10 minutes of $\mathrm{F}-18 \mathrm{NaF}$ administration, in addition to conventional PET/CT imaging which is performed 30-45 minutes after F-18 NaF injection $(2,16)$. An early-phase F-18 NaF PET/CT scan is known to provide the same information that can be acquired with the perfusion- and blood-pool phase images of the three-phase bone scintigraphy, showing increased radiotracer uptake in the lesion with regional hyperemia (2). Because metastatic bone lesions have high density of vasculature, metastatic bone lesions revealed increased radiotracer uptake on earlyphase images of the three-phase bone scintigraphy and dualphase F-18 NaF PET/CT in previous case reports (17-20). Considering the findings of these previous studies, F-18 $\mathrm{NaF}$ PET/CT with additional acquisition of early-phase images may help with the diagnosis of bone metastasis. However, in previously published literature, no studies have investigated the clinical value of dual-phase F-18 NaF
PET/CT in detecting bone metastasis.

Therefore, in the present study, we retrospectively enrolled patients with malignancies who had undergone dual-phase F-18 NaF PET/CT scan for evaluating a solitary bone lesion on bone scintigraphy and investigated whether dual-phase PET/CT could enhance the diagnostic ability to detect bone metastasis compared to conventional F-18 NaF $\mathrm{PET} / \mathrm{CT}$.

\section{Methods}

\section{Study population}

We retrospectively reviewed the electronic medical records of 148 patients with histopathologically diagnosed malignant diseases who underwent F-18 NaF PET/CT for diagnosing bone metastasis in our medical center between January 2015 and December 2018. Among these patients, a total of 113 patients ( 80 men and 33 women; age range of 39-84 years) were finally enrolled in the present study according to the following inclusion criteria: patients (I) who showed a solitary bone lesion suggestive of potential bone metastasis on staging Tc-99m methylene diphosphonate bone scintigraphy or a newly appeared solitary bone lesion suggestive of potential bone metastasis on surveillance bone scintigraphy without a previous history of bone metastasis, (II) who had undergone dual-phase F-18 $\mathrm{NaF}$ PET/CT for the differential diagnosis of the solitary bone lesion seen on bone scintigraphy, and (III) who had a histopathological confirmation of a bone lesion or had undergone follow-up imaging examinations for a clinical diagnosis of the bone lesion. We excluded the patients (I) who had a history of bone metastasis, (II) who were shown to have multifocal bone lesions suggestive of potential bone metastasis on bone scintigraphy, (III) in whom an early phase F-18 NaF PET/CT scan was not performed, (IV) in whom the final diagnosis of the bone lesion could not be determined, and $(\mathrm{V})$ that had undergone any treatment for bone metastasis between their bone scintigraphy and PET/ CT scan. The median interval between bone scintigraphy and F-18 NaF PET/CT was 17 days (range, 1-61 days).

This retrospective study was approved by the Institutional Review Board of our medical center (IRB 
number: 2020-03-026). The study protocol was performed in accordance with the ethical standards laid down in the Declaration of Helsinki. Informed consent for dual-phase F-18 NaF PET/CT was obtained from the all patients enrolled in the present study before PET/CT scan.

\section{Dual-phase F-18 NaF PET/CT scan}

An F-18 NaF PET/CT scan was performed with a dedicated PET/CT scanner (Biograph mCT 128 scanner, Siemens Healthcare, Knoxville, TN, USA). A dual-phase F-18 NaF PET/CT scan consists of an early-phase scan and a conventional scan with both phase scans performed with static imaging as described in our previous study (2). There were no special patient preparations before PET/CT scanning. For the early-phase PET/CT scan, the anatomical parts of patients in which the solitary bone lesion were observed on bone scintigraphy were placed at the center of the field of view. Immediately after intravenous injection of $185 \mathrm{MBq}$ of F-18 NaF, early-phase PET/CT images were acquired. Initially, a noncontrast-enhanced CT scan was performed at $100 \mathrm{~mA}$ and $120 \mathrm{kVp}$ with a slice thickness of $5 \mathrm{~mm}$. Subsequently, a PET scan was performed in one or two bed positions at 2 minutes for bed position using a three-dimensional acquisition mode. After the earlyphase PET/CT scan, a conventional PET/CT scan was performed approximately 45 minutes after the F-18 NaF injection. The conventional PET/CT scan was performed from the vertex to the proximal femur or from the vertex to the feet according to the clinical condition of the patient using the same setting as in the early-phase scan. All PET images were reconstructed using point-spread-function modeling and time-of-flight reconstruction with attenuation correction.

\section{F-18 NaF PET/CT image analysis}

All dual-phase F-18 NaF PET/CT images were retrospectively reviewed by four nuclear medicine physicians and two quantitative parameters, the lesion-to-blood pool uptake ratio and the lesion-to-bone uptake ratio, were measured. Two nuclear medicine physicians (JWL and SJJ) visually assessed only the conventional PET/CT images and measured the lesion-to-bone uptake ratio. The other two nuclear medicine physicians (YJP and SML) visually evaluated the dual-phase PET/CT scan images and measured the lesion-to-blood pool uptake ratio. Discrepancies between the two readers were resolved by discussion in order to reach a consensus. Only primary tumor sites and bone scintigraphy images performed before the PET/CT were provided to the nuclear medicine physicians who were blinded to any other clinical information.

On visual analysis of the conventional scan images, suspected bone lesions corresponding to solitary bone lesions on bone scintigraphy were interpreted as positive or negative for bone metastasis. Bone lesions that showed intense radiotracer uptake and were considered unrelated to benign processes were determined to be positive bone lesions. Negative bone lesions were defined as lesions in which radiotracer uptake was not intense, located around the joint area, or lesions related to benign processes, such as enthesopathies, degenerative changes, or iatrogenic lesions. For visual analysis of the dual-phase PET/CT images, conventional PET/CT images were evaluated first; for the bone lesions that were positive on conventional PET/CT images, early-phase scan images were further assessed. On early-phase scans, a positive bone lesion was defined as a bone lesion with increased radiotracer uptake similar to or greater than the uptake of the blood pool. Bone lesions that showed positive uptake on both early-phase and conventional scans images were classified as positive bone lesion on dual-phase PET/CT.

For quantitative analysis, the lesion-to-blood pool uptake ratio on early-phase images and the lesion-tobone uptake ratio on conventional scan images were measured. The standardized uptake value (SUV) was used as a descriptive indicator for the degree of uptake by the bone lesion. On both early-phase and bone-phase PET/ CT images, a spheroid-shaped volume-of-interest (VOI) was manually drawn for the suspected bone lesion, and the maximum SUV of the suspected bone lesion was calculated. For measuring reference organ uptake, the mean SUVs of the blood pool uptake on early-phase scan images and the normal bone uptake on conventional scan images were calculated. For analysis of blood pool uptake, VOIs were manually drawn over the artery, and the arterial wall containing calcified plaques was excluded from the VOIs. For normal bone uptake, three VOIs were manually drawn over the vertebral bodies of the thoracic and lumbar spines and the mean SUV of those three VOIs were calculated. Using the maximum SUV of the bone lesion and mean SUV of blood pool and normal bone, the lesion-to-blood pool uptake ratio and lesion-to-bone uptake ratio were calculated for each suspected bone lesion. 


\section{Standard of reference}

All patient clinical information, including the results of physical, laboratory, imaging, and histopathological examinations, were obtained from their electronic medical records. In patients who had undergone bone biopsy, the final diagnosis was made by histopathological evaluation. In patients who had not undergone bone biopsy, an imagingbased diagnosis was made using follow-up imaging studies that were performed at least 1 year after the PET/CT scan. Patients who demonstrated signs of progression of their bone lesion, osteolytic lesions changing into osteosclerotic lesions during treatment, or an increased number of bone lesions suggestive of potential bone metastasis on follow-up imaging examinations were considered to have metastatic bone lesions. A multidisciplinary team consisting of three experienced board-certified physicians who did not participate in the PET/CT imaging analysis reviewed medical records and follow-up imaging examinations of patients, making the final clinical diagnosis of suspected bone lesions without knowing the dual-phase PET/CT results. According to the final diagnosis, all patients were categorized into two groups: patients with metastatic bone lesions and patients with nonmetastatic bone lesions.

\section{Statistical analysis}

Based on the results of the visual image analysis, the sensitivity, specificity, positive predictive value, negative predictive value, and accuracy of the visual analysis of conventional and dual-phase PET/CT for detecting bone metastasis were determined. The diagnostic accuracies of four parameters of PET/CT (visual analysis and lesionto-bone uptake ratio on conventional PET/CT and visual analysis and lesion-to-blood pool uptake ratio on dual-phase PET/CT scans) were evaluated on the basis of the area under the receiver operating characteristic (ROC) curve (AUC) values. With the optimal cut-off values determined by the ROC curve analysis, the sensitivity, specificity, positive predictive value, negative predictive value, and accuracy of lesion-to-blood pool uptake ratio and lesion-tobone uptake ratio were assessed. On comparing AUC values between parameters, a Bonferroni correction was performed to adjust for multiple comparisons. Mann-Whitney test was performed to compare differences in the lesion-to-blood pool uptake ratios on early-phase scans and lesion-to-bone uptake ratios on conventional scans between metastatic bone lesions and nonmetastatic bone lesions. The Kruskal-
Wallis test with post-hoc comparisons was performed to compare the lesion-to-blood pool uptake ratio and lesionto-bone uptake ratio according the dual-phase PET/CT findings and CT findings. MedCalc Statistical Software (version 19.2, MedCalc Software, Ostend, Belgium) was used for all statistical tests. A $\mathrm{P}$ value of less than 0.05 was considered statistically significant.

\section{Results}

\section{Patient characteristics}

The characteristics of the 113 enrolled patients are summarized in Table 1 . The most common site of primary cancer lesions in enrolled patients was prostate $(62.8 \%)$, followed by breast (20.4\%). No enrolled patients had any previous history of bone metastasis or metabolic bone disease or any other recent major traumatic events. Of all patients, $38(33.6 \%)$ had a solitary bone lesion on initial staging bone scintigraphy, and the remaining 75 (65.4\%) had a newly appeared solitary bone lesion on surveillance bone scintigraphy during or after treatment. Most of the solitary bone lesions suggestive of potential bone metastasis were located in spine, pelvic bone, or sacrum.

Of the enrolled patients, metastatic bone lesions were diagnosed in 28 patients $(24.8 \%)$ and the remaining 85 patients $(75.2 \%)$ were diagnosed with nonmetastatic bone lesions. The most common site of bone metastasis was pelvic bone (10 patients, $35.7 \%$ ), followed by spine (7 patients, $25.0 \%$ ), rib (5 patients, $17.9 \%$ ), sacrum (4 patients, $14.3 \%$ ), femur (1 patient, $3.6 \%$ ), and scapula (1 patient, $3.6 \%$ ). Among the 28 patients with metastatic bone lesions, 6 patients $(21.4 \%)$ were diagnosed by bone biopsy and the diagnosis of the remaining 22 patients (78.6\%) was made by follow-up imaging studies. On CT images of F-18 NaF PET/CT, 16 metastatic bone lesions (57.1\%) showed osteolytic features, 6 lesions $(21.4 \%)$ osteosclerotic features, 3 lesions (10.7\%) mixed features, and the remaining 3 lesions (10.7\%) showed no abnormal findings.

\section{Diagnostic ability of conventional PET/CT}

The results of the visual analysis of F-18 NaF PET/ CT images are shown in Table 2. On visual analysis of conventional PET/CT, all 28 metastatic bone lesions were considered to be positive bone lesions, resulting in a sensitivity of $100 \%$ (Figure 1). Meanwhile, there were 25 
Table 1 Patient demographics $(\mathrm{n}=113)$

\begin{tabular}{|c|c|}
\hline Characteristics & Number of patients (\%) \\
\hline Age & 67 years $\left(39-84\right.$ years) ${ }^{\dagger}$ \\
\hline \multicolumn{2}{|l|}{ Sex } \\
\hline Men & $80(70.8)$ \\
\hline Women & $33(29.2)$ \\
\hline \multicolumn{2}{|l|}{ Primary tumor } \\
\hline Prostate cancer & $71(62.8)$ \\
\hline Breast cancer & $23(20.4)$ \\
\hline Renal cell carcinoma & $7(6.2)$ \\
\hline Urothelial carcinoma & $3(2.7)$ \\
\hline Gastric cancer & $3(2.7)$ \\
\hline Others $^{\ddagger}$ & $6(5.3)$ \\
\hline \multicolumn{2}{|c|}{ Reason for bone scintigraphy } \\
\hline Initial staging & $38(33.6)$ \\
\hline Surveillance & $75(65.4)$ \\
\hline \multicolumn{2}{|c|}{ Anatomical sites of single bone lesion } \\
\hline Spine & $35(31.0)$ \\
\hline Pelvic bone & $29(25.7)$ \\
\hline Sacrum & $17(15.0)$ \\
\hline Rib & $14(12.4)$ \\
\hline Femur & $8(7.1)$ \\
\hline Sternum & $6(5.3)$ \\
\hline Skull & $2(1.8)$ \\
\hline Scapula & $1(0.9)$ \\
\hline Clavicle & $1(0.9)$ \\
\hline
\end{tabular}

${ }^{\dagger}$, expressed as median (range); ${ }^{\ddagger}$, Consisted of one patient with thymic cancer, one with rectal cancer, one with pancreatic cancer, one with lung cancer, one with gallbladder cancer, and one with malignant gastrointestinal stromal tumor.

false-positive bone lesions identified on visual analysis of conventional PET/CT images among the 85 nonmetastatic bone lesions. The visual analysis of conventional PET/CT showed a sensitivity of $100.0 \%$ (95\% CI, $87.7-100.0 \%$ ), specificity of $70.6 \%$ (95\% CI, $59.7-80.0 \%$ ), positive predictive value of $52.8 \%$ (95\% CI, 44.6-60.9\%), negative predictive value of $100.0 \%$, (95\% CI, 93.5-100.0\%), and accuracy of $77.9 \%$ (Table 3). Among 28 patients with metastatic bone lesions, F-18 NaF PET/CT detected additional metastatic bone lesions in 7 patients $(25.0 \%)$
Table 2 The results of the visual analysis of dual-phase F-18 NaF bone PET/CT scan for detecting bone metastasis in 113 patients

\begin{tabular}{lcc}
\hline & \multicolumn{2}{c}{ Final diagnosis } \\
\cline { 2 - 3 } & Bone metastasis & $\begin{array}{c}\text { Nonmetastatic bone } \\
\text { lesion }\end{array}$ \\
\hline Conventional PET/CT & 28 & 25 \\
Positive & 0 & 60 \\
Negative & & \\
Dual-phase PET/CT & 27 & 10 \\
Positive & 1 & 75 \\
Negative &
\end{tabular}

F-18 NaF, F-18 sodium fluoride; PET/CT, positron emission tomography/computed tomography.

which were not observed on bone scintigraphy (Figure 1).

On ROC curve analysis, the optimal cut-off value was 2.50 for the lesion-to-bone uptake ratio on the conventional scan. Using a cut-off value of 2.50 , the lesion-to-bone uptake ratio on conventional scans showed a sensitivity of 92.9\% (95\% CI, 76.5-99.1\%), specificity of $42.4 \%$ (95\% CI, 31.7-53.6\%), positive predictive value of $34.7 \%$ (95\% CI, 30.1-59.5\%), negative predictive value of $94.7 \%$ (95\% CI, $82.2-98.6 \%$ ), and accuracy of $54.9 \%$ (Table 3).

\section{Diagnostic ability of dual-phase PET/CT}

Of 28 metastatic bone lesions, the visual analysis of dualphase PET/CT images detected 27 lesions, resulting in a sensitivity of $96.4 \%$ (Table 2). One patient with prostate cancer showed a false-negative finding on visual analysis of the dual-phase PET/CT images (Figure 2). Compared to the results of the visual analysis of conventional PET/ CT images, the number of patients with false-positive findings was significantly reduced with the visual analysis of dual-phase PET/CT $(\mathrm{P}=0.005)$. The 15 lesions that were considered false-positive lesions on conventional PET/CT, but negative lesions on dual-phase PET/CT were located in the spine (5 lesions; Figure 3), rib (4 lesions), pelvic bone (2 lesions), skull (1 lesion), sternum (1 lesion), sacrum (1 lesion), and femur (1 lesion). The sites of primary cancers in those 15 lesions were the prostate ( 8 patients), breast (5 patients), kidney (1 patient), and thymus (1 patient). The final diagnosis of the 15 lesions were degenerative lesions (7 lesions), compression fractures or post-traumatic lesions (7 lesions), and benign bone tumor (1 lesion). Those 10 
A

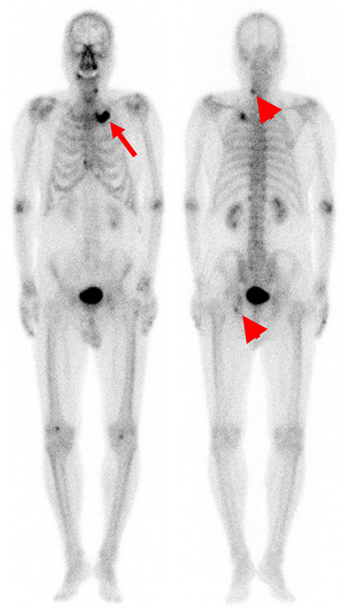

B

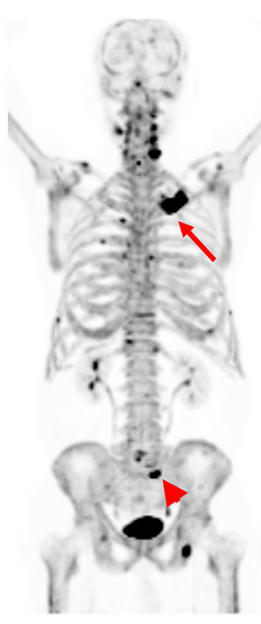

C

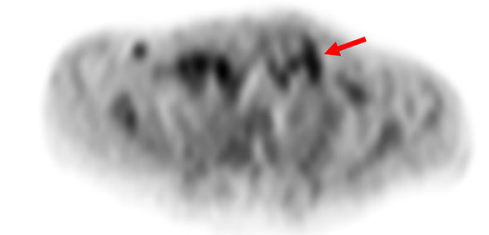

$\mathrm{E}$

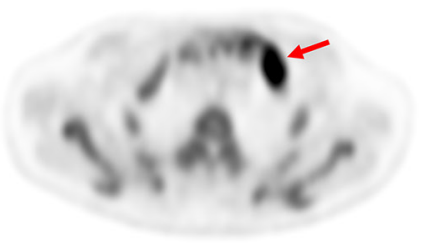

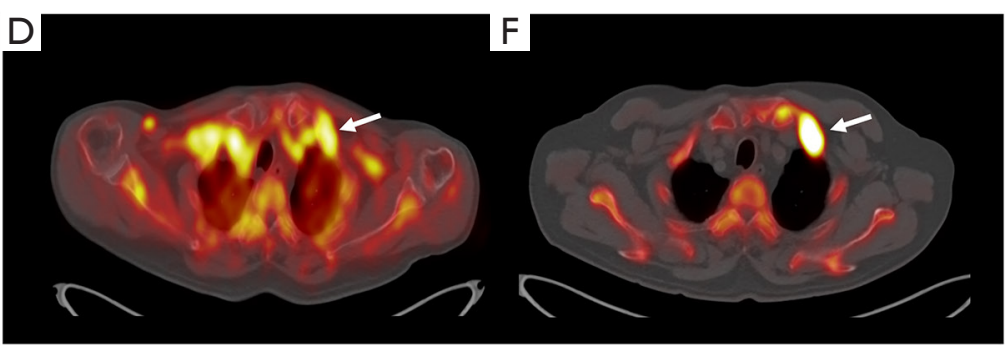

Figure 1 Bone scintigraphy (A) and transaxial PET (C) and fused PET/CT (D) from the early-phase scan and a maximum intensity projection image (B) and transaxial PET (E) fused PET/CT (F) images from the conventional scan in F-18 NaF PET/CT of a 78-yearold man with prostate cancer. During clinical follow-up after starting hormone treatment, he underwent surveillance bone scintigraphy because of his increased serum prostate-specific antigen level $(24.55 \mathrm{ng} / \mathrm{mL})$. On bone scintigraphy images (A), focally increased radiotracer uptake newly appears in his left 1st rib (arrow in A), while focally increased radiotracer uptake in the lower cervical spine and left acetabulum (arrowheads in A) is seen in a previous bone scintigraphy, and considered degenerative bone lesions. Both early-phase and conventional scan images show intensely increased radiotracer uptake at the left 1st rib (arrows in B-F) with a lesion-to-blood pool uptake ratio of 0.94, suggesting metastatic bone lesion. Furthermore, another focally increased radiotracer uptake is observed in the sacrum (arrowhead in B), suggesting another metastatic bone lesion. The left rib lesion was histopathologically confirmed to be a metastatic bone lesion from prostate cancer. PET/CT, positron emission tomography/computed tomography.

Table 3 Diagnostic accuracy of the visual analysis, lesion-to-blood blood uptake ratio on early-phase scan, and lesion-to-bone uptake ratio on convention scan

\begin{tabular}{|c|c|c|c|c|}
\hline Diagnostic ability & \multicolumn{2}{|c|}{ Early-phase scan } & \multicolumn{2}{|c|}{ Convention scan } \\
\hline Cut-off value & - & 0.76 & - & 2.50 \\
\hline Sensitivity (\%), (95\% Cl) & $96.4(81.7-100.0)$ & $92.9(76.5-99.1)$ & $100.0(87.7-100.0)$ & $92.9(76.5-99.1)$ \\
\hline Specificity (\%), (95\% Cl) & $88.2(79.4-94.2)$ & $81.2(71.2-88.8)$ & $70.6(59.7-80.0)$ & $42.4(31.7-53.6)$ \\
\hline NPV (\%), (95\% Cl) & $98.7(91.6-100.0)$ & $97.2(90.0-99.2)$ & $100.0(93.5-100.0)$ & 94.7 (82.2-98.6) \\
\hline Accuracy (\%) & 90.3 & 83.2 & 77.9 & 54.9 \\
\hline AUC (95\% Cl) & $0.923(0.858-0.965)$ & $0.917(0.850-0.960)$ & $0.853(0.774-0.913)$ & $0.756(0.666-0.832)$ \\
\hline
\end{tabular}

PET/CT, positron emission tomography/computed tomography; PPV, positive predictive value; NPV, negative predictive value; AUC, area under the receiver operating characteristic curve.

lesions that still showed false-positive findings on dualphase PET/CT images were clinically diagnosed with degenerative lesions, compression fractures or posttraumatic lesions on follow-up imaging studies (Figure 4).
The visual analysis of dual-phase PET/CT showed a sensitivity of $96.4 \%$ (95\% CI, $81.7-100.0 \%)$, specificity of $88.2 \%$ (95\% CI, 79.4-94.2\%), positive predictive value of 73.0\% (95\% CI, 60.0-82.9\%), negative predictive value 
A

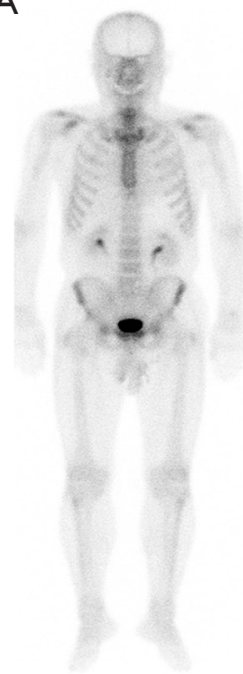

B

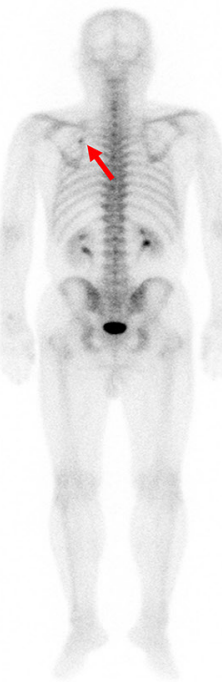

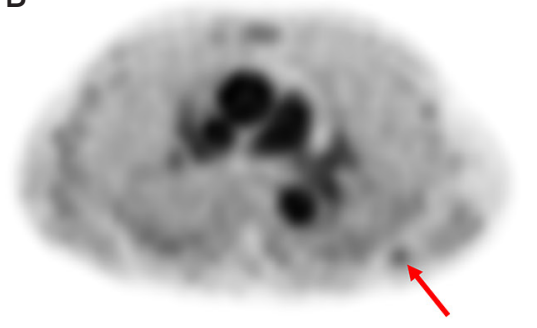

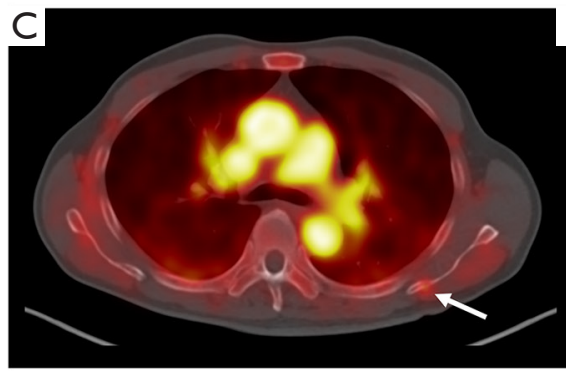

D
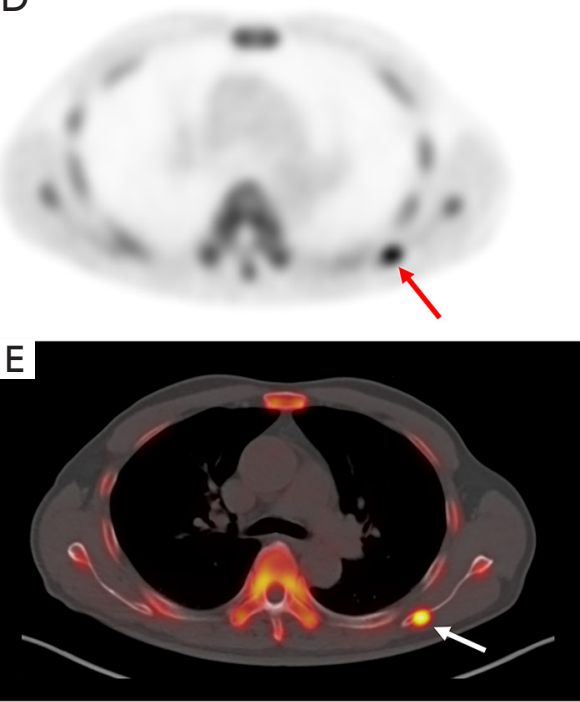

Figure 2 Bone scintigraphy (A) and transaxial PET (B) and fused PET/CT (C) from the early-phase scan and transaxial PET (D) and fused PET/CT (E) from the conventional scan in F-18 NaF PET/CT of a 72-year-old man with prostate cancer. Staging bone scintigraphy images show focally increased radiotracer uptake in the left scapula (arrow on A) with serum prostate-specific antigen level of $55.70 \mathrm{ng} / \mathrm{mL}$. Bone-phase scan images show focal intensely increased radiotracer uptake in the left scapula (arrows on D and E); however, only mildly increased radiotracer uptake is observed on early-phase scan images (arrows on B and C) with a lesion-to-blood pool uptake ratio of 0.43 . The lesion was clinically diagnosed as being a metastatic bone lesion. PET/CT, positron emission tomography/computed tomography.

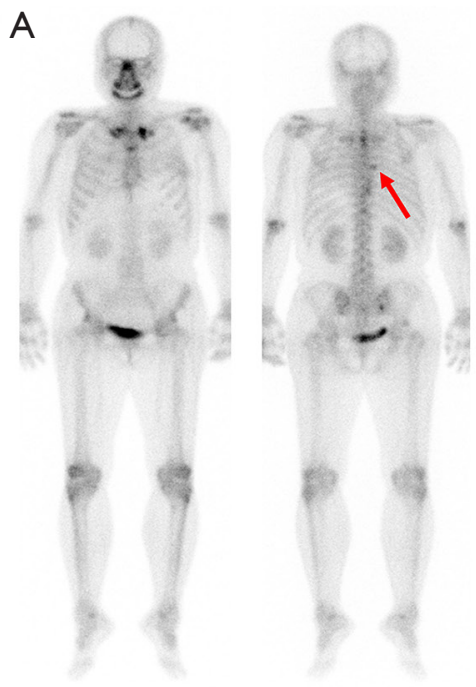

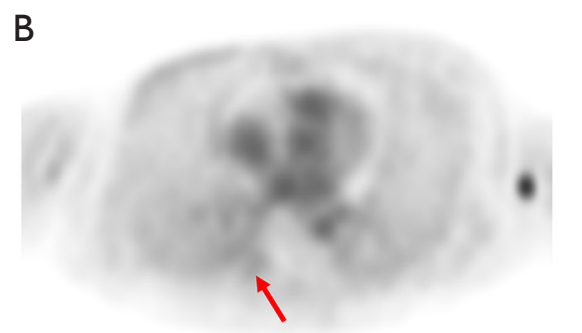

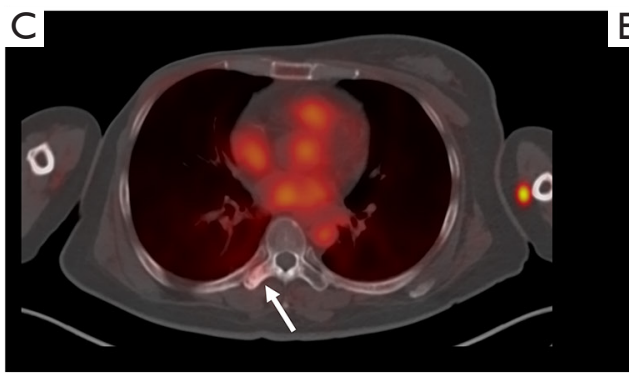

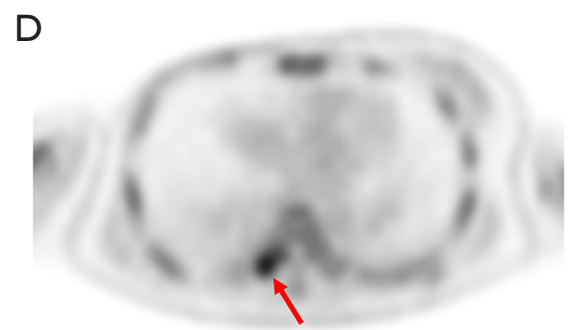

E

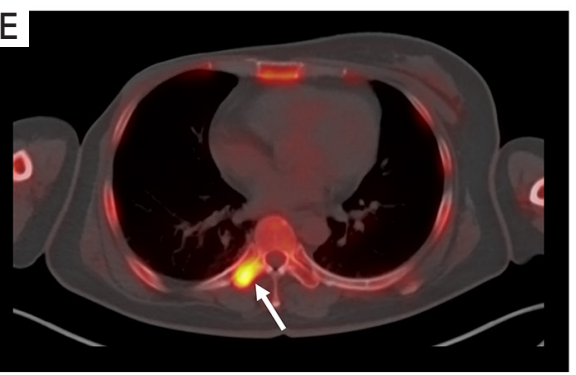

Figure 3 Bone scintigraphy (A) and transaxial PET (B) and fused PET/CT (C) from the early-phase scan and transaxial PET (D) and fused PET/CT (E) from the conventional scan in F-18 NaF PET/CT of a 47-year-old woman with breast cancer. On surveillance bone scintigraphy after right mastectomy, focally increased radiotracer uptake is newly observed in the T7 spine (arrow in A). On bone-phase scan images, focal intensely increased radiotracer uptake is shown in the right transverse process of T7 spine (arrows on D and E), but only minimal radiotracer uptake was shown on early-phase scan images (arrows on B and C) with a lesion-to-blood pool uptake ratio of 0.29. On follow-up imaging studies, the bone lesion was clinically diagnosed as nonmetastatic bone lesion. PET/CT, positron emission tomography/ computed tomography. 
A

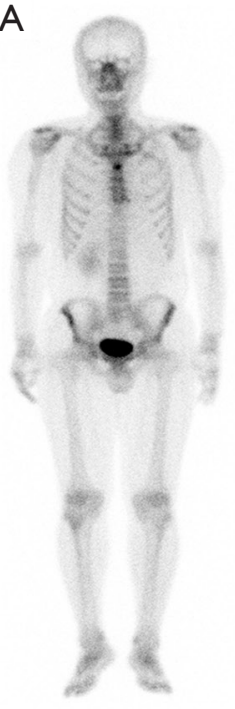

B

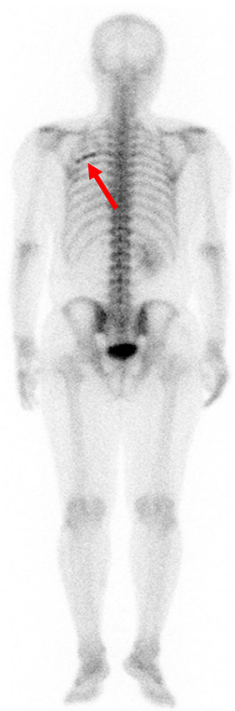

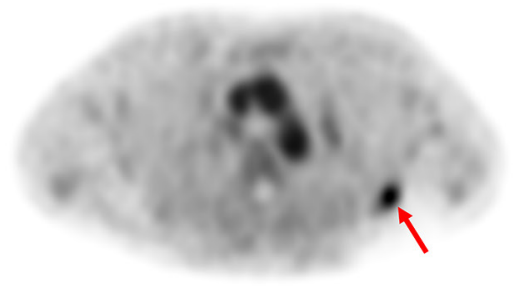

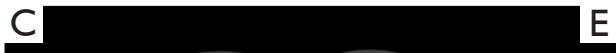

D

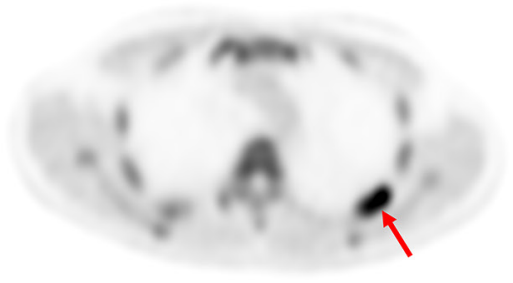

E

Figure 4 Bone scintigraphy (A) and transaxial (B) and fused PET/CT (C) from the early-phase scan and transaxial PET (D) and fused PET/CT (E) from the conventional scan in F-18 NaF PET/CT of a 39-year-old man with renal cell carcinoma. On surveillance bone scintigraphy performed after curative surgical resection, an elongated rib lesion is shown (arrow on A). The rib lesion shows intensely increased radiotracer uptake on early-phase scan images (arrows on B and C) with a lesion-to-blood pool uptake ratio of 1.48 as well as on bone-phase scan images (arrows on D and E). The rib lesion was clinically diagnosed as nonmetastatic post-traumatic bone lesion by followup imaging studies. PET/CT, positron emission tomography/computed tomography.

of $98.7 \%$ (95\% CI, 91.6-100.0\%), and accuracy of $90.3 \%$ (Table 3). Because the additional metastatic bone lesions detected by F-18 NaF PET/CT were not included in the scan fields of early-phase scans in all 7 patients, those lesions could not be evaluated by early-phase scan images.

On the ROC curve analysis, using an optimal cut-off value of 0.76 , the lesion-to-blood pool uptake ratio on early-phase scan showed a sensitivity of $92.9 \%$ (95\% CI, $76.5-99.1 \%$ ), specificity of $81.2 \%$ (95\% CI, 71.2-88.8\%), positive predictive value of $61.9 \%$ (95\% CI, 50.8-71.9\%), negative predictive value of $97.2 \%$ (95\% CI, 90.0-99.2\%), and accuracy of $83.2 \%$ (Table 3).

\section{Comparison of quantitative parameters}

In comparisons of quantitative parameters between metastatic and nonmetastatic bone lesions, 28 patients with metastatic bone lesions showed significantly higher values of both the lesion-to-blood pool uptake ratio on early-phase scan $(1.59 \pm 0.89$ vs. $0.60 \pm 0.31)$ and the lesionto-bone uptake ratio on conventional scan $(6.57 \pm 3.58$ vs. $3.65 \pm 2.57)$ than 85 patients with nonmetastatic bone lesions $(\mathrm{P}<0.001$ for all; Figure 5$)$. Of the 28 metastatic bone lesions, 20 lesions (71.4\%) had lesion-to-blood pool uptake ratios $>1.0$. In comparison of quantitative parameters according to the findings of dual-phase PET/CT (Table 4), the Kruskal-Wallis test revealed significant differences of both quantitative parameters among the groups $(\mathrm{P}<0.001$ for all). On post-hoc analysis, 28 patients with metastatic bone lesions and 10 patients with false-positive lesions on dual-phase PET/CT had significantly higher values of the lesion-to-blood pool uptake ratio and lesion-to-bone uptake ratio than 15 patients with false-positive lesions only on conventional PET/CT and 60 patients with negative findings on conventional PET/CT $(\mathrm{P}<0.05)$. In comparison of quantitative parameters of 28 metastatic bone lesions according to the CT findings, there were no significant differences of either quantitative parameters between lesions with osteosclerotic features, osteolytic features, mixed features, and no abnormal CT findings by the KruskalWallis test $(\mathrm{P}>0.05$; Table 4$)$.

\section{Comparison of ROC curves}

On the ROC curve analysis, the results of the visual analysis of dual-phase PET/CT images revealed the highest AUC 

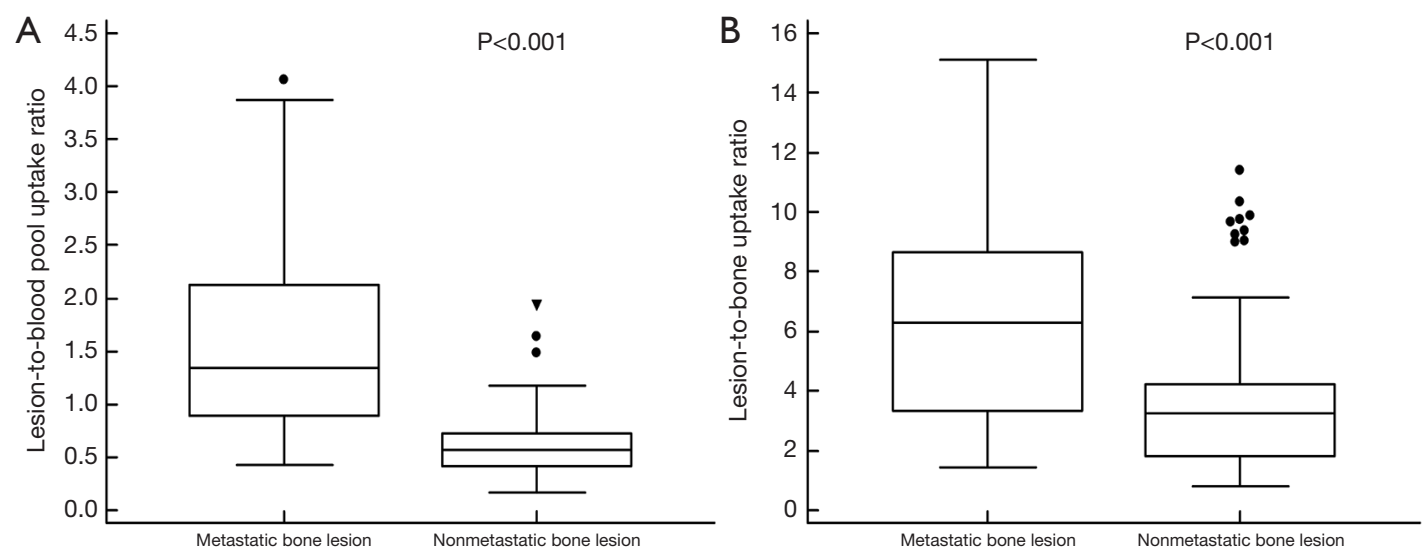

Figure 5 Distribution of the lesion-to-blood pool uptake ratio on an early-phase scan (A) and the lesion-to-bone uptake ratio on a conventional scan (B) in 28 metastatic bone lesions and 85 nonmetastatic bone lesions.

Table 4 The lesion-to-blood pool uptake ratio on early-phase scan and lesion-to-bone uptake ratio on conventional scan according to the findings of F-18 NaF PET/CT

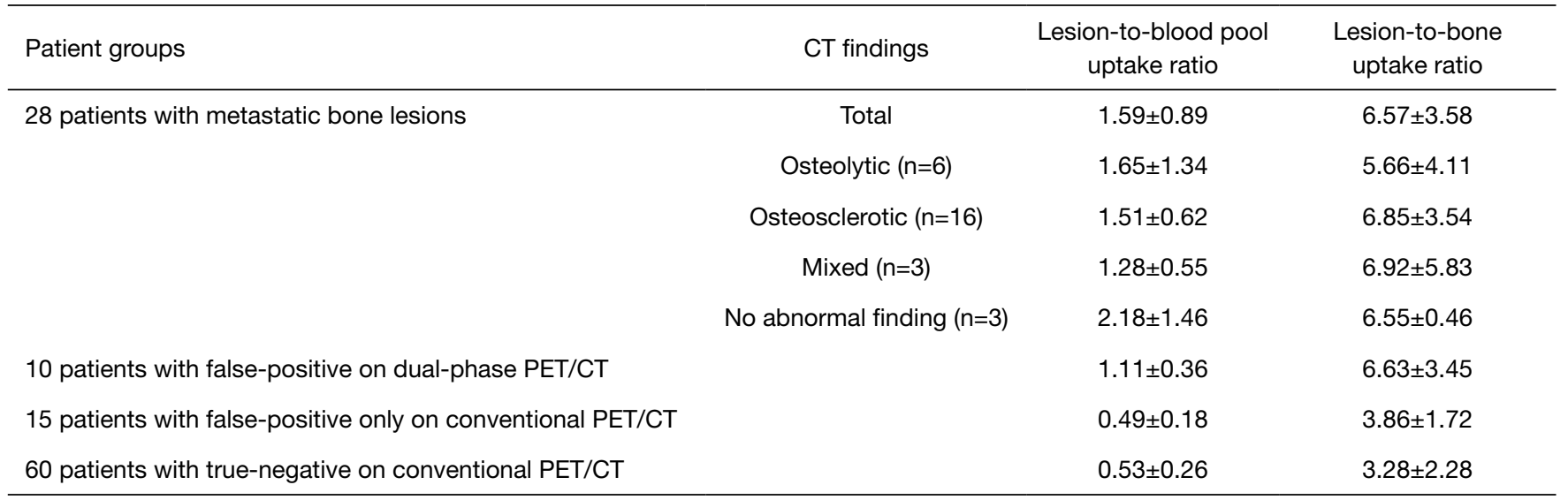

F-18 NaF, F-18 sodium fluoride; PET/CT, positron emission tomography/computed tomography.

value $(0.923 ; 95 \% \mathrm{CI}, 0.858-0.965)$, followed by the lesionto-blood pool uptake ratio in early-phase scans (AUV value, 0.917 ; $95 \% \mathrm{CI}, 0.850-0.960)$, the results of the visual analysis of conventional PET/CT images (AUC value, 0.853; 95\% CI, 0.774-0.913), and the lesion-to-bone uptake ratio in conventional scans (AUC value, 0.756; 95\% CI, 0.666-0.832; Table 3; Figure 6). In the pairwise comparisons of AUC values between parameters of F-18 NaF PET/ CT images after Bonferroni correction, the results of the visual analysis of the dual-phase PET/CT images showed significantly higher AUC values than the results of the visual analysis of conventional $\mathrm{PET} / \mathrm{CT}$ images $(\mathrm{P}=0.040)$ and the lesion-to-bone uptake ratio in conventional scans $(\mathrm{P}=0.003)$. Moreover, the lesion-to-blood pool uptake ratio in early-phase scans showed significantly higher AUC values than the lesion-to-bone uptake ratio in conventional scans $(\mathrm{P}=0.004)$. In contrast, other comparisons of AUC values failed to reveal any statistical significance $(\mathrm{P}>0.05)$.

\section{Discussion}

In this study, using a dual-phase protocol, we were able to improve the diagnostic ability of F-18 NaF PET/CT for diagnosing bone metastasis. Dual-phase F-18 NaF PET/CT was first introduced in 2014 for clinical use in patients with chronic osteomyelitis (13). Afterwards, several studies have shown the clinical value of dual-phase PET/CT imaging for evaluating surgical site infections following orthopedic 


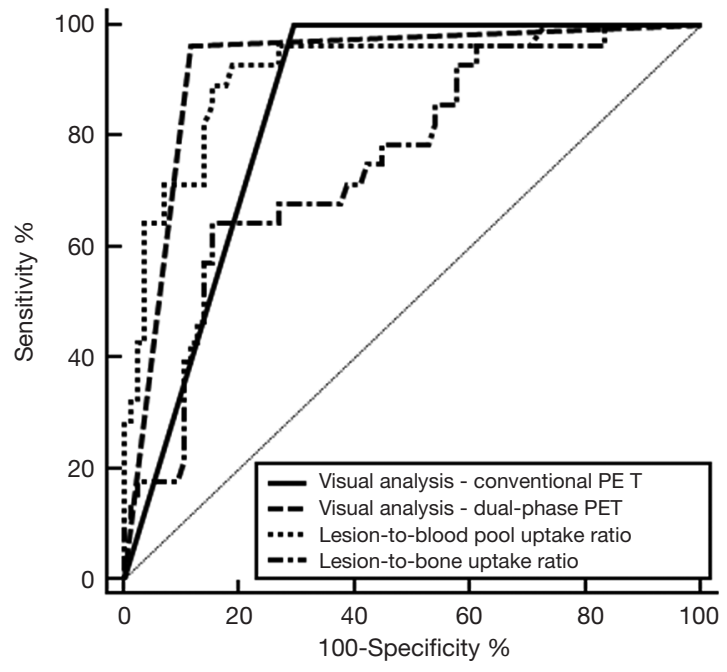

Figure 6 Comparison of receiver operating characteristic curves for the visual analysis of conventional PET/CT (conventional PET) and dual-phase PET/CT (dual-phase PET), the lesion-toblood pool uptake ratio on the early-phase scan, and the lesionto-bone uptake ratio on the conventional scan. PET/CT, positron emission tomography/computed tomography.

surgery, septic hip joints, and sacroiliitis (2,14,21-23). Since the degree of radiotracer uptake in early-phase scan images can reflect the regional hyperemia of a lesion, dualphase F-18 NaF PET/CT has been shown to have a high diagnostic ability in the context of infectious inflammation and active sacroiliitis $(2,14,23)$. In metastatic bone lesions, metastatic cancer cells stimulate angiogenesis by various tumor-derived growth factors to support the growth of the lesion, resulting in increased vascular structure densities $(20,24)$. Our previous case report documented the case of a breast cancer patient who showed increased radiotracer uptake in early-phase F-18 NaF PET/CT images in metastatic bone lesions that was subsequently decreased after chemotherapy, suggesting that dual-phase scans might have a clinical role in diagnosing and evaluating treatment responses of bone metastasis (19). The present study assessed the clinical use of dual-phase F-18 NaF PET/CT imaging protocols for bone metastases and showed that $71.4 \%$ of metastatic bone lesions had higher radiotracer uptake than the blood pool on early-phase scan images, indicating increased perfusion and blood pool in metastatic bone lesions. With the help of early-phase scan findings, dual-phase F-18 NaF PET/CT showed high sensitivity and specificity for diagnosing bone metastasis.

Solitary bone metastasis is a very rare clinical condition, accounting for only $2-3 \%$ of all malignant spread situations in the skeletal system (25). Although the spine and pelvic bone are the most common sites for solitary bone metastases and solitary bone metastases in the rib, skull, and extremities remain uncommon (25-29), the lesion site has no clinical value when differentiating metastatic bone lesions from benign bone lesions (30). Although planar bone scintigraphy has a high sensitivity for detecting bone metastasis with examining the whole-body skeleton, the ability of bone scintigraphy to differentiate metastatic bone lesions from benign bone lesions remains limited $(31,32)$. Therefore, diverse imaging examinations, including singlephoton emission computed tomography (SPECT)/CT, F-18 NaF PET/CT, F-18 fluorodeoxyglucose (FDG) PET/ $\mathrm{CT}$, and magnetic resonance imaging (MRI), have been used to improve the diagnostic accuracy for bone metastasis in patients with abnormal findings on bone scintigraphy (10,30,32-34). However, for patients who showed solitary bone lesions on bone scintigraphy, only a small number of studies have investigated the clinical value of the aforementioned imaging methods. SPECT/CT intrinsically had an inferior sensitivity and resolution than did F-18 NaF PET/CT images; however, previous studies demonstrated that SPECT/CT could improve the specificity for detecting bone metastasis and reduce the number of equivocal bone lesions $(7,11,35,36)$. In previous studies comparing the diagnostic ability of FDG PET/CT with bone scintigraphy, most false-positive lesions on FDG PET/CT consisted of solitary bone lesions, and there was no significant difference in terms of the accuracy of diagnosis of solitary bone metastasis between them $(32,37)$. Furthermore, another study with solitary bone lesions revealed the limited diagnostic accuracy for FDG PET/CT in differentiating metastasis among rib lesions (69.2\%) (30). In terms of MRI, because only a few studies focused on the assessment of solitary bone lesion, its diagnostic ability cannot be properly estimated (34). In terms of F-18 NaF PET/CT, the present study was the first study to evaluate its diagnostic accuracy in the context of a solitary bone lesion. Similar to the results of studies in patients with multiple bone lesions (7-9), our study also revealed a high sensitivity of $100.0 \%$ for conventional PET/CT imaging in diagnosing bone metastasis in patients with solitary bone lesions. Meanwhile, a moderate specificity of $70.6 \%$ and accuracy of $77.9 \%$ were shown, which were quite a bit lower than the results of previous studies. Considering that solitary bone lesions on F-18 NaF PET/CT had often been categorized as inconclusive bone lesions in previously published studies 
$(6,7,9)$, conventional F-18 NaF PET/CT images could have a limited value in differentiating metastatic bone lesions from benign bone lesions in the context of solitary bone lesions. On the other hand, the specificity and accuracy of dual-phase F-18 NaF PET/CT increased to $88.2 \%$ and $90.3 \%$, respectively, suggesting that F-18 NaF PET/CT with the dual-phase protocol could help with the differential diagnosis of bone metastasis. Along with other known imaging methods, dual-phase PET/CT could be considered another imaging method option for assessment of solitary bone lesions.

In contrast, the present study also revealed several limitations inherent to dual-phase F-18 NaF PET/CT. Since traumatic and inflammatory bone lesions are also accompanied by regional hyperemia, those bone lesions can still show false-positive findings on dual-phase PET/CT images $(2,23,38)$, which limit the positive predictive value of dual-phase PET/CT. Furthermore, as shown in Figure 4, a metastatic bone lesion can show only mild radiotracer uptake in early-phase scan images, which would eventually lower the sensitivity of the scan compared to conventional PET/CT. Above all, dual-phase PET/CT can be performed only for a patient in whom the region of the target bone lesion can be selected, because early-phase scanning cannot cover the whole-body skeleton. Therefore, rather than being incorporated into routine clinical use, the selected use of dual-phase imaging protocol might be more beneficial in a context as shown in the present study.

Although one of the advantages of F-18 NaF PET/ CT, as compared with bone scintigraphy, lies in its ability to quantitatively measure radiotracer uptake, the clinical values of the quantitative parameters of F-18 NaF PET/CT remain undefined $(2,16,39)$. In our study, we also measured two quantitative parameters, the lesion-to-blood pool uptake ratio on early-phase scan and the lesion-to-bone uptake ratio on bone-phase scan, and compared their diagnostic ability to the results of the visual analysis. On comparing quantitative parameters, metastatic bone lesions were shown to have significant higher values of both parameters than did nonmetastatic bone lesions. However, given that the uptake levels of metastatic bone lesions overlapped with nonmetastatic bone lesions and that the location and pattern of uptake are also considered in the interpretation of F-18 NaF PET/CT images (7), the characterization of bone lesions solely based on quantitative parameters would have limited value. Accordingly, the present study showed that the quantitative parameter in each phase scan had a lower AUC value for diagnosing bone metastasis than the results of the visual analysis. Nevertheless, considering the significant differences in the lesion-to-bone uptake ratios according to the findings of dual-phase PET/CT scan, the quantitative parameters measured on F-18 NaF PET/CT may play an auxiliary role in the characterization of bone lesions. Further studies are warranted to clarify their role.

This study has a number of unaddressed limitations. First, because a relatively small number of patients, with a broad age range, an unequal sex distribution, and selected from a single medical center, were enrolled in the study, the results need to be further validated. Moreover, due to its retrospective design, we were not able to investigate whether dual-phase PET/CT can lead to changes in treatment planning as compared to conventional PET/CT. Second, because only the patients who showed a solitary bone lesion on bone scintigraphy were enrolled in the study, the proportion of patients with metastatic bone lesions were relatively low, which might cause misunderstanding of clinical significance of dual-phase F-18 NaF PET/CT. Third, since SPECT/CT is known to have high diagnostic accuracy for detecting bone metastasis and to show similar ability to F-18 NaF PET/CT in reducing the equivocal lesions on bone scintigraphy $(11,35,36)$, a future study that compares the diagnostic ability between SPECT/CT and dual-phase PET/CT is needed. Another limitation is that various types of cancer were included in the study, thereby leading increased clinical heterogeneity and inevitably affecting the results. Finally, histopathological confirmation, the current gold standard to prove a bone metastasis, was not performed in most metastatic bone lesions. However, performing a biopsy in every patient is impractical, and follow-up imaging studies have been commonly used as the standard of reference in other studies $(40,41)$.

\section{Conclusions}

In the present study, conventional F-18 NaF PET/CT showed a sensitivity of $100 \%$ in the context of diagnosing bone metastasis in cancer patients with solitary bone lesions and detecting additional bone metastases in $25.0 \%$ of patients with metastatic bone lesions. However, because of the significant portion of patients with false-positive findings, conventional PET/CT was shown to have only moderate specificity and accuracy. Dual-phase F-18 NaF PET/CT showed both high sensitivity and specificity, improving the diagnostic accuracy to $90.3 \%$, and the visual analysis of dual-phase PET/CT was shown to have the highest AUC value of 0.923 for diagnosing bone metastasis. 
Dual-phase F-18 NaF PET/CT might be helpful for differentiating metastatic bone lesions in patients with malignancies who had been shown to have a solitary bone lesion on bone scintigraphy.

\section{Acknowledgments}

Funding: This work was supported by Soonchunhyang University Research Fund and the National Research Foundation of Korea (NRF) grant funded by the Korea government (Ministry of Science and ICT) (grant number: NRF-2017R1C1B5075905).

\section{Footnote}

Conflicts of Interest: All authors have completed the ICMJE uniform disclosure form (available at http://dx.doi. org/10.21037/qims-20-607). The authors have no conflicts of interest to declare.

Ethical Statement: This retrospective study was approved by the Institutional Review Board of Soonchunhyang University Cheonan Hospital (IRB number: 2020-03026), and the study protocol was performed in accordance with the ethical standards laid down in the Declaration of Helsinki. Informed consent for dual-phase F-18 NaF $\mathrm{PET} / \mathrm{CT}$ was obtained from the all patients enrolled in the present study before the PET/CT scan.

Open Access Statement: This is an Open Access article distributed in accordance with the Creative Commons Attribution-NonCommercial-NoDerivs 4.0 International License (CC BY-NC-ND 4.0), which permits the noncommercial replication and distribution of the article with the strict proviso that no changes or edits are made and the original work is properly cited (including links to both the formal publication through the relevant DOI and the license). See: https://creativecommons.org/licenses/by-nc-nd/4.0/.

\section{References}

1. Bastawrous S, Bhargava P, Behnia F, Djang DS, Haseley DR. Newer PET application with an old tracer: role of $18 \mathrm{~F}-\mathrm{NaF}$ skeletal PET/CT in oncologic practice. Radiographics 2014;34:1295-316.

2. Lee JW, Yu SN, Yoo ID, Jeon MH, Hong CH, Shim JJ, Chang SH, Lee SM. Clinical application of dual-phase F-18 sodium-fluoride bone PET/CT for diagnosing surgical site infection following orthopedic surgery. Medicine (Baltimore) 2019;98:e14770.

3. Blake GM, Puri T, Siddique M, Frost ML, Moore AEB, Fogelman I. Site specific measurements of bone formation using [(18)F] sodium fluoride PET/CT. Quant Imaging Med Surg 2018;8:47-59.

4. Jassel IS, Siddique M, Frost ML, Moore AEB, Puri T, Blake GM. The influence of CT and dual-energy X-ray absorptiometry (DXA) bone density on quantitative [(18)F] sodium fluoride PET. Quant Imaging Med Surg 2019;9:201-9.

5. Grant FD, Fahey FH, Packard AB, Davis RT, Alavi A, Treves ST. Skeletal PET with 18F-fluoride: applying new technology to an old tracer. J Nucl Med 2008;49:68-78.

6. Even-Sapir E, Metser U, Flusser G, Zuriel L, Kollender Y, Lerman H, Lievshitz G, Ron I, Mishani E. Assessment of malignant skeletal disease: initial experience with 18F-fluoride PET/CT and comparison between 18F-fluoride PET and 18F-fluoride PET/CT. J Nucl Med 2004;45:272-8.

7. Abikhzer G, Srour S, Fried G, Drumea K, Kozlener E, Frenkel A, Israel O, Fogelman I, Kagna O. Prospective comparison of whole-body bone SPECT and sodium $18 \mathrm{~F}$-fluoride PET in the detection of bone metastases from breast cancer. Nucl Med Commun 2016;37:1160-8.

8. Sheikhbahaei S, Jones KM, Werner RA, Salas-Fragomeni RA, Marcus CV, Higuchi T, Rowe SP, Solnes LB, Javadi MS. (18)F-NaF-PET/CT for the detection of bone metastasis in prostate cancer: a meta-analysis of diagnostic accuracy studies. Ann Nucl Med 2019;33:351-61.

9. Rao L, Zong Z, Chen Z, Wang X, Shi X, Yi C, Zhang X. 18F-labeled $\mathrm{NaF}$ PET-CT in detection of bone metastases in patients with preoperative lung cancer. Medicine (Baltimore) 2016;95:e3490.

10. Poulsen MH, Petersen H, Hoilund-Carlsen PF, Jakobsen JS, Gerke O, Karstoft J, Steffansen SI, Walter S. Spine metastases in prostate cancer: comparison of technetium99m-MDP whole-body bone scintigraphy, [(18) F] choline positron emission tomography(PET)/computed tomography (CT) and [(18) F]NaF PET/CT. BJU Int 2014;114:818-23.

11. Löfgren J, Mortensen J, Rasmussen SH, Madsen C, Loft A, Hansen AE, Oturai P, Jensen KE, Mork ML, Reichkendler M, Hojgaard L, Fischer BM. A prospective study comparing $(99 \mathrm{~m})$ Tc-hydroxyethylene-diphosphonate planar bone scintigraphy and whole-body SPECT/ CT with (18)F-fluoride PET/CT and (18)F-fluoride PET/MRI for diagnosing bone metastases. J Nucl Med 
2017;58:1778-85.

12. Zacho HD, Fonager RF, Nielsen JB, Haarmark C, Hendel HW, Johansen MB, Mortensen JC, Petersen LJ. Observer agreement and accuracy of (18)F-sodium fluoride PET/

CT in the diagnosis of bone metastases in prostate cancer. J Nucl Med 2020;61:344-9.

13. Freesmeyer M, Stecker FF, Schierz JH, Hofmann GO, Winkens T. First experience with early dynamic (18) F-NaF-PET/CT in patients with chronic osteomyelitis. Ann Nucl Med 2014;28:314-21.

14. Sawicki LM, Lutje S, Baraliakos X, Braun J, Kirchner J, Boos J, Heusch P, Ruhlmann V, Herrmann K, Umutlu L, Quick HH, Antoch G, Buchbender C. Dual-phase hybrid (18) F-Fluoride Positron emission tomography/MRI in ankylosing spondylitis: Investigating the link between MRI bone changes, regional hyperaemia and increased osteoblastic activity. J Med Imaging Radiat Oncol 2018;62:313-9.

15. Velez EM, Desai B, Jadvar H. Treatment response assessment of skeletal metastases in prostate cancer with (18)F-NaF PET/CT. Nucl Med Mol Imaging 2019;53:247-52.

16. Segall G, Delbeke D, Stabin MG, Even-Sapir E, Fair J, Sajdak R, Smith GT. SNM practice guideline for sodium 18F-fluoride PET/CT bone scans 1.0. J Nucl Med 2010;51:1813-20.

17. Çayir D, Bozkurt M, Gultekin SS, Karacalioglu AO. Earlier phases of bone scintigraphy can better delineate the extent of soft tissue involvement of bone metastasis. Clin Nucl Med 2018;43:854-6.

18. Siraj QH, Ali M, Hussain A, Rafi CM, Ahmed M. Threephase skeletal scintigraphy in a solitary lytic osseous metastasis of renal cell carcinoma. J Pak Med Assoc 1990;40:192-4.

19. Seo JH, Lee SM, Yu SN, Lee JW, Lee JE. Clinical usefulness of two-phase (18)F-sodium-fluoride ((18) $\mathrm{F}-\mathrm{NaF}$ ) bone PET/CT for evaluating treatment response of bone metastases from breast cancer: Case report. Rev Esp Med Nucl Imagen Mol 2019;38:238-42.

20. Raymaekers K, Stegen S, van Gastel N, Carmeliet G. The vasculature: a vessel for bone metastasis. Bonekey Rep 2015;4:742.

21. Shim JJ, Lee JW, Jeon MH, Lee SM. Recurrent surgical site infection of the spine diagnosed by dual (18)F-NaFbone PET/CT with early-phase scan. Skeletal Radiol 2016;45:1313-6.

22. Moon JH, Lee SM, Hong CH, Lee JW. Early evaluation of inflammatory focus and treatment response using
18F-sodium fluoride bone positron emission tomography/ computed tomography in patients with metallic implants: a case report. J Korean Soc Radiol 2018;78:408-11.

23. Kumar R, Kumar R, Kumar V, Malhotra R. Comparative analysis of dual-phase 18F-fluoride PET/CT and three phase bone scintigraphy in the evaluation of septic (or painful) hip prostheses: A prospective study. J Orthop Sci 2016;21:205-10.

24. Nyangoga H, Mercier P, Libouban H, Basle MF, Chappard D. Three-dimensional characterization of the vascular bed in bone metastasis of the rat by microcomputed tomography (MicroCT). PLoS One 2011;6:e17336.

25. Rubin P, Brasacchio R, Katz A. Solitary metastases: illusion versus reality. Semin Radiat Oncol 2006;16:120-30.

26. Ugras N, Yalcinkaya U, Akesen B, Kanat O. Solitary bone metastases of unknown origin. Acta Orthop Belg 2014;80:139-43.

27. Tumeh SS, Beadle G, Kaplan WD. Clinical significance of solitary rib lesions in patients with extraskeletal malignancy. J Nucl Med 1985;26:1140-3.

28. Hashmi R, Uetani M, Ogawa Y, Aziz A. Clinical significance of a solitary hot spot in the skull. Nucl Med Commun 1999;20:703-10.

29. Peng H, Zhang L, Zhou T, Li W, Li W, Ma L, Zhang R. Characterization of solitary lesions in the extremities on whole-body bone scan in patients with known cancer: Contribution of single-photon emission computed tomography/computed tomography. Front Oncol. 2019;9:607.

30. Lim CH, Ahn TR, Moon SH, Cho YS, Choi JY, Kim BT, Lee KH. PET/CT features discriminate risk of metastasis among single-bone FDG lesions detected in newly diagnosed non-small-cell lung cancer patients. Eur Radiol 2019;29:1903-11.

31. Zhang Y, Shi H, Cheng D, Jiang L, Xiu Y, Li B, Gu $\mathrm{Y}$, Chen S. Added value of SPECT/spiral CT versus SPECT in diagnosing solitary spinal lesions in patients with extraskeletal malignancies. Nucl Med Commun 2013;34:451-8.

32. Kim MR, Roh JL, Kim JS, Choi SH, Nam SY, Kim SY. 18F-fluorodeoxyglucose-positron emission tomography and bone scintigraphy for detecting bone metastases in patients with malignancies of the upper aerodigestive tract. Oral Oncol 2008;44:148-52.

33. Zhang Y, Li B, Shi H, Yu H, Gu Y, Xiu Y. Added value of SPECT/spiral CT versus SPECT or CT alone in diagnosing solitary skeletal lesions. Nuklearmedizin 2017;56:139-45. 
34. Smolarz K, Jungehulsing M, Krug B, Linden A, Gohring UJ, Schicha H. Magnetic resonance tomography of the bone marrow in cancer patients with a solitary area of increased uptake in the bone scintigram. Nuklearmedizin 1990;29:269-73.

35. Fonager RF, Zacho HD, Langkilde NC, Fledelius J, Ejlersen JA, Haarmark C, Hendel HW, Lange MB, Jochumsen MR, Mortensen JC, Petersen LJ. Diagnostic test accuracy study of (18)F-sodium fluoride PET/CT, (99m)Tc-labelled diphosphonate SPECT/CT, and planar bone scintigraphy for diagnosis of bone metastases in newly diagnosed, high-risk prostate cancer. Am J Nucl Med Mol Imaging 2017;7:218-27.

36. Palmedo H, Marx C, Ebert A, Kreft B, Ko Y, Türler A, Vorreuther R, Göhring U, Schild HH, Gerhardt T, Pöge U, Ezziddin S, Biersack HJ, Ahmadzadehfar H. Wholebody SPECT/CT for bone scintigraphy: diagnostic value and effect on patient management in oncological patients. Eur J Nucl Med Mol Imaging 2014;41:59-67.

37. Hur J, Yoon CS, Ryu YH, Yun MJ, Suh JS. Accuracy of fluorodeoxyglucose-positron emission tomography for diagnosis of single bone metastasis: comparison with bone scintigraphy. J Comput Assist Tomogr 2007;31:812-9.

38. Querellou S, Moineau G, Le Duc-Pennec A, Guillo P, Turzo A, Cotonea Y, Le Nen D, Salaun PY. Detection of occult wrist fractures by quantitative radioscintigraphy: a prospective study on selected patients. Nucl Med Commun 2009;30:862-7.

39. Muzahir S, Jeraj R, Liu G, Hall LT, Rio AM, Perk T, Jaskowiak C, Perlman SB. Differentiation of metastatic vs degenerative joint disease using semi-quantitative analysis with (18)F-NaF PET/CT in castrate resistant prostate cancer patients. Am J Nucl Med Mol Imaging 2015;5:162-8.

40. Broos WAM, van der Zant FM, Wondergem M, Knol RJJ. Accuracy of $18 \mathrm{~F}-\mathrm{NaF}$ PET/CT in bone metastasis detection and its effect on patient management in patients with breast carcinoma. Nucl Med Commun 2018;39:325-33.

41. Shen CT, Qiu ZL, Han TT, Luo QY. Performance of 18F-fluoride PET or PET/CT for the detection of bone metastases: a meta-analysis. Clin Nucl Med 2015;40:103-10.
Cite this article as: Lee JW, Park YJ, Jeon YS, Kim KH, Lee JE, Hong SH, Lee SM, Jang SJ. Clinical value of dual-phase F-18 sodium fluoride PET/CT for diagnosing bone metastasis in cancer patients with solitary bone lesion. Quant Imaging Med Surg 2020;10(11):2098-2111. doi: 10.21037/qims-20-607 